\title{
Article \\ Caenorhabditis elegans Multi-Tracker Based on a Modified Skeleton Algorithm
}

\author{
Pablo E. Layana Castro (D), Joan Carles Puchalt (D), Antonio García Garví (D) and Antonio-José Sánchez-Salmerón * \\ Instituto de Automática e Informática Industrial, Universitat Politècnica de València, 46022 Valencia, Spain; \\ pablacas@doctor.upv.es (P.E.L.C.); juapucro@doctor.upv.es (J.C.P.); angar25a@etsid.upv.es (A.G.G.) \\ * Correspondence: asanchez@isa.upv.es
}

Citation: Layana Castro, P.E.; Puchalt, J.C.; García Garví, A.; Sánchez-Salmerón, A.-J. Caenorhabditis elegans Multi-Tracker Based on a Modified Skeleton Algorithm. Sensors 2021, 21, 5622. https:/ /doi.org/10.3390/s21165622

Academic Editor: Christophoros Nikou

Received: 13 July 2021

Accepted: 18 August 2021

Published: 20 August 2021

Publisher's Note: MDPI stays neutral with regard to jurisdictional claims in published maps and institutional affiliations.

Copyright: (c) 2021 by the authors. Licensee MDPI, Basel, Switzerland. This article is an open access article distributed under the terms and conditions of the Creative Commons Attribution (CC BY) license (https:/ / creativecommons.org/licenses/by/ $4.0 /)$.
Abstract: Automatic tracking of Caenorhabditis elegans (C. egans) in standard Petri dishes is challenging due to high-resolution image requirements when fully monitoring a Petri dish, but mainly due to potential losses of individual worm identity caused by aggregation of worms, overlaps and body contact. To date, trackers only automate tests for individual worm behaviors, canceling data when body contact occurs. However, essays automating contact behaviors still require solutions to this problem. In this work, we propose a solution to this difficulty using computer vision techniques. On the one hand, a skeletonization method is applied to extract skeletons in overlap and contact situations. On the other hand, new optimization methods are proposed to solve the identity problem during these situations. Experiments were performed with 70 tracks and 3779 poses (skeletons) of $C$. elegans. Several cost functions with different criteria have been evaluated, and the best results gave an accuracy of $99.42 \%$ in overlapping with other worms and noise on the plate using the modified skeleton algorithm and $98.73 \%$ precision using the classical skeleton algorithm.

Keywords: C. elegans assays; lifespan; healthspan; image detection; multi-tracker; standard Petri dishes

\section{Introduction}

The nematode Caenorhabditis elegans (C. elegans) is a widely studied animal model, as its diverse age-related behavioral patterns provide valuable information on the function of its nervous system and is, therefore, an attractive model to evaluate the effects of mutations [1]. This facilitates the study and treatment of aging, as well as age-related pathologies and neurodegenerative disorders in humans at advanced ages [2,3]. Many of these studies have shown that automatic tracking applications based on computer vision systems help to reduce the manual cost of data acquisition and research hours, improving potential observation of the effects of drug trials [4] and improvements in lifespan or "shelflife" [5-7]. These systems provide quantitative information on alterations in the individual motility and behavior of worms produced by chemical substances in their environment (chemotaxis), providing statistical data that allow further research in the field of health and wellness.

C. elegans demonstrate group behavior [8-10], among the best known are courtship, mating, aggression, rearing and foraging. Group behavior assays are currently being performed, for example, research into the effect of $\mathrm{O}_{2}$ in food search analysis, and aggregation [11,12]. These assays, like others, are visualized manually, due to the complexity of solving the identification problem during an overlapping or body contact of these worms. Currently, the automatic [13-21] or semi-automatic applications discard the data from tracks where there are these particular cases (overlapping and bodies contacts) [22-24]. Overlapping can take place among worms or may also be due to plate noise. Plate noise was defined as segmentation errors due to edges, or opaque waste in the plate.

Certain applications use diverse techniques and methods such as length [25], smoothness [26,27], previous segmentation [28], or other complex methods [29-34] to solve track- 
ing problems during aggregation. The aforementioned methods were tested by our team and results indicated that previous segmentation is one of the most significant criteria to help identify worms within an aggregation. This was taken as a starting point to design different trackers, adding and combining numerous criteria, and it was found that the criteria of completeness and color used with the improvement of the form of skeletonizing [35] can help to identity the worms in the next image. Furthermore, we introduced three new criteria: length, smoothness, and noise, revealing that they can be useful in re-identification.

We present different multi-trackers which, unlike any other trackers, are fully automatic (Table 1). Segmentation and identification of the edge, the interior rim of the Petri dish (area of interest), and segmentation tracks made by worms are performed without the intervention of human operators. These new methods show worm-tracking accuracy of above $98 \%$ in nematode aggregations and problems related to plate noise. The best result reaches $99.42 \%$ accuracy.

Table 1. Comparison with other multi-trackers. This table shows the comparison of our multi-tracker with respect to others (tierpsy-tracker [22], WF-NTP.v3 [23]).

\begin{tabular}{|c|c|c|c|c|}
\hline \multicolumn{5}{|c|}{ Comparative Table } \\
\hline Name & $\begin{array}{l}\text { Illumination } \\
\text { Technique }\end{array}$ & Features & Method & Situations solved \\
\hline $\begin{array}{l}\text { Tierpsy } \\
\text { Tracker } \\
{[22]}\end{array}$ & $\begin{array}{l}\text { Standard } \\
\text { backlight }\end{array}$ & $\begin{array}{l}\text { Manual } \\
\text { parameter } \\
\text { setting }\end{array}$ & $\begin{array}{l}\text { Skeletons, out- } \\
\text { lines and seg- } \\
\text { mentations }\end{array}$ & Individual tracking \\
\hline $\begin{array}{l}\text { WF- } \\
\text { NTP.v3 } \\
{[23]}\end{array}$ & $\begin{array}{l}\text { Flat-field il- } \\
\text { lumination }\end{array}$ & $\begin{array}{l}\text { Manual } \\
\text { parameter } \\
\text { setting }\end{array}$ & $\begin{array}{l}\text { Skeletons and } \\
\text { centroids }\end{array}$ & Individual tracking \\
\hline Ours & $\begin{array}{l}\text { Active back- } \\
\text { light system } \\
{[36,37]}\end{array}$ & $\begin{array}{l}\text { Fully auto- } \\
\text { matic }\end{array}$ & $\begin{array}{l}\text { Improved skele- } \\
\text { ton and segmen- } \\
\text { tations }\end{array}$ & $\begin{array}{l}\text { Individual tracking, } \\
\text { overlaps, body con- } \\
\text { tacts, rolled worms } \\
\text { and occlusions. }\end{array}$ \\
\hline
\end{tabular}

\section{Materials and Methods}

\subsection{Nematode Strains and Culture}

The strains (N2) and CB1370 daf-2 (e1370) used in the study were provided by the University of Minnesota Caenorhabditis Genetics Center. The C. elegans were kept at $20{ }^{\circ} \mathrm{C}$ and cultured on $55 \mathrm{~mm}$ diameter NGM plates with $1 \mu \mathrm{g} / \mathrm{mL}$ of fungizone to prevent fungal contamination [38]. Escherichia coli (E. coli) strain OP50 was used as standard prey for C. elegans in the laboratory. FUdR $(0.2 \mathrm{mM})$ was used to prevent replication. The cultured plates were 10, 15, 30, 60 and 100 adult-young worms synchronized from worm eggs incubated at $20^{\circ} \mathrm{C}$. This variety in the number of worms per plate allowed to obtain aggregations of two C. elegans or more.

\subsection{Proposed Tracking Method}

At present, automatic or semi-automatic trackers discard the data of tracks where there are overlap or body contacts, due to the difficulty of solving the identity of each individual in these situations. Our method does solve these situations using a fully automated pipeline based on an intelligent active backlight, a modified skeletonization method and new criteria to solve the final optimization problem.

Steps of the proposed tracking method are described in Figure 1a-f, and more detailed in Sections 2.3-2.7, respectively. This method starts with the acquisition of sequences of C. elegans images, Figure 1a. These image sequences are then processed (Figure 1b) to obtain mathematical models of C. elegans Figure 1c, and possible solutions to these in an 
aggregation. Finally, the result (Figure 1f) is the optimal minimization cost between models and possible solutions.

a

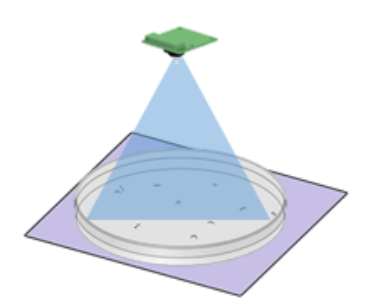

b

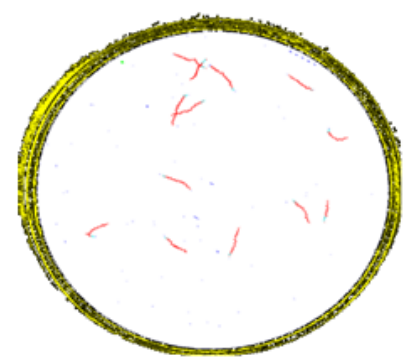

C
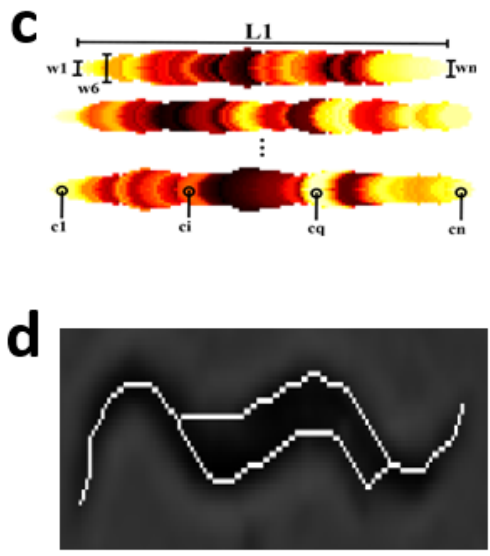

e
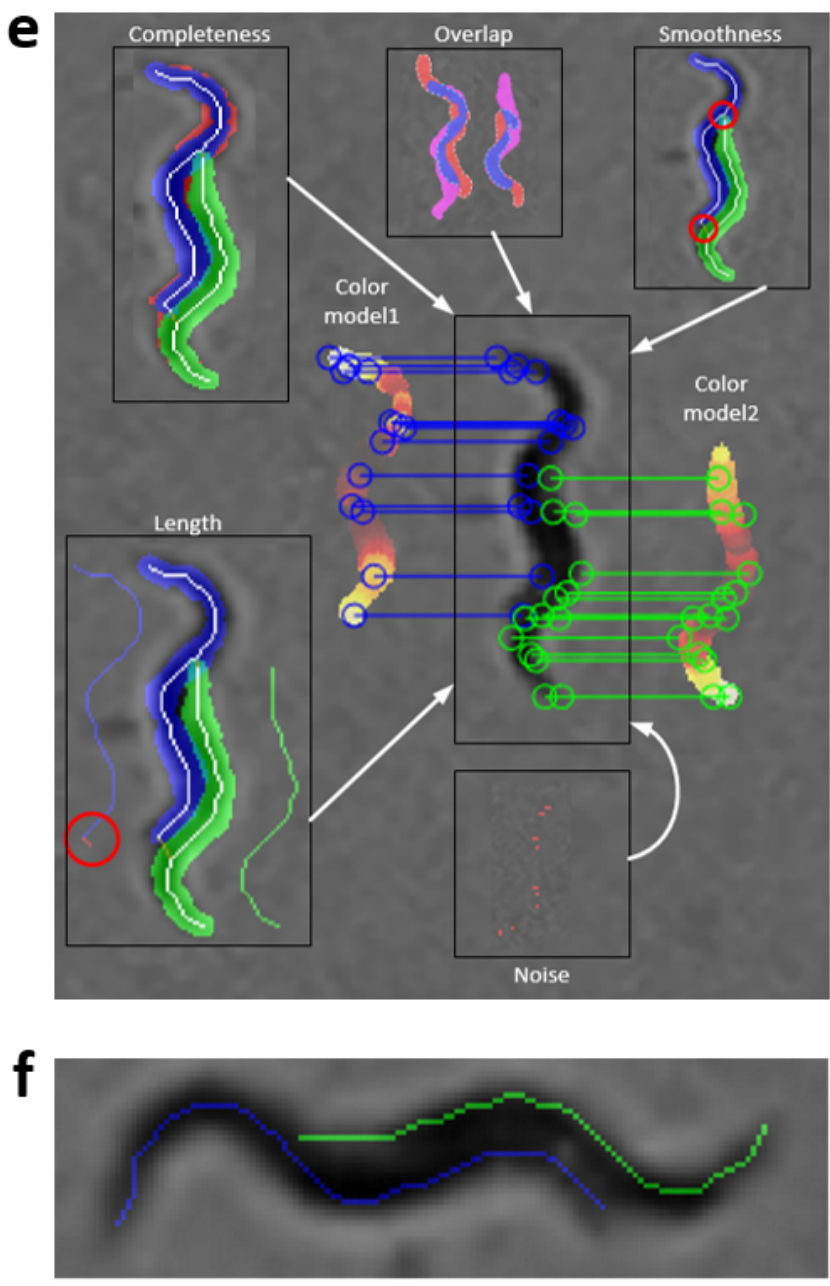

Figure 1. General scheme of image processing. The image shows the different stages that the tracker goes through to obtain the results of the skeletons and to follow all the worms within the plate. (a) Image acquisition [37]. (b) Pre-processing image. (c) Worm models. (d) Improved skeleton using proposed method [35]. (e) Optimization method. (f) Optimization results (predicted poses).

\subsection{Image Acquisition}

The image acquisition process was performed using the capture system [37]. To use this system, first, a laboratory operator removed the plates from the incubator, then the plates were analyzed to find condensation on the covers, if so, they were removed, otherwise, the image sequence was captured. The system [37] is automatic and captures an image every second. Escherichia coli (E. coli) strain OP50 was placed in the center of the plate to capture the worms inside the Petri dish and not scaling the edges or near them. The intelligent active backlighting method was used as the illumination technique [36]. This method is more robust than standard backlighting methods, as it allows us to obtain constant intensity values for the bottom of the Petri dish and the worms (greater than 48 and less than 35, respectively). This facilitated automatic segmentation with fixed thresholds on all images.

The image sequence was acquired at a resolution of $1944 \times 1944$ pixels and a frequency of $1 \mathrm{~Hz}$ (1 image per second) using the system [37] (Table 2). 
Table 2. Components of the data acquisition system.

\begin{tabular}{lll}
\hline & & \multicolumn{2}{c}{ System Components } \\
\hline $\mathbf{N}^{\circ}$ & Name & Description \\
\hline 1 & Raspberry Pi V3 b+ & Procesador: 64-bit ARM Cortex-A53, 1.4 GHz \\
& RAM Size: 1 GB LPDDR2 SDRAM \\
\hline \multirow{3}{*}{2} & Sensor: OmniVision OV5647 \\
& & Pixel resolution: $2592 \times 1944$ \\
& \multirow{2}{*}{ Raspberry Pi Camera V1.3 } & Pixel size: $1.4 \times 1.4 \mu \mathrm{m}$ \\
& & Field of view: $53.50^{\circ} \times 41.41^{\circ}$ \\
& & Optical size: $1 / 4^{\prime \prime}$ \\
& & Focal length: 2.9 \\
\hline \multirow{3}{*}{3} & \multirow{2}{*}{ Raspberry Pi display } & Screen display size: $7^{\prime \prime}$ \\
& & Resolution: $800 \times 480$ and $60 \mathrm{fps}$ \\
& & Color: 24-bit RGB colour \\
\hline
\end{tabular}

This image acquisition system is open hardware and its assembly procedure, parts and description are described in detail in another work [37]. Worm tracking, using these image acquisition conditions, is a very challenging problem. An image resolution of $1944 \times 1944$ pixels is the lowest able to detect worms, when a complete Petri dish ( $55 \mathrm{~mm}$. of diameter) is monitored with a fixed camera. In addition, this problem was solved by using a low frame rate of $1 \mathrm{~Hz}$. The dataset collected was composed by sequences of 30 images where contact events between worms occurred, to perform all the experiments.

\subsection{Image Processing}

Image processing began with the segmentation of the region of interest and C. elegans tracks in the image sequence (white circle and red track in Figure 1b). To obtain the region of interest, first, a segmentation was carried out on all the images of the sequence using a threshold with a fixed intensity value (35 in the gray scale). Then, an AND operation was executed between all the segmented images, the result obtained went through a "Fillhole" operation to fill small holes. The region of interest was selected as the largest connected component of the resulting image. It is important to mention that the use of a fixed threshold for all images is due to the intelligent active backlighting method as a lighting technique [36], as this system allows to conserve background intensity values constantly.

In parallel to the previous step, the $C$. elegans tracks were segmented with a process using different threshold levels Figure 1b. Threshold levels were below 35 on the gray scale. The resulting segmentations went through two filters in order to eliminate those tracks that did not correspond to worms. In the first stage, those tracks with an area smaller than the minimum area of a worm were filtered. The second filter analyzed the skeleton of each image, if the skeleton did not correspond to a minimum expected length, it was classified as noise. The results of the number of skeletons found in each image were stored in a $30 \times \mathrm{N}$ matrix, where $\mathrm{N}$ is total number of tracks and 30 is the number of images in the sequence.

Due to low resolution of the worms, a scale factor of 3 was applied to increase resolution. This process was applied by obtaining the model to the end of tracking. The model of each worm was obtained by analyzing $30 \times \mathrm{N}$ matrix, finding image " $\mathrm{k}$ " of 30 images, where worms were further apart from each other, and their ends, head-tail, were also separate. Tracking of each worm started from image $k+1$ to image 30 and from image $\mathrm{k}-1$ to the first. The skeletonization method proposed in previous work [35] was used in each image. This method used distance transformation [39] to obtain possible worm skeletons, and through of an optimization method using different criteria found the best skeleton prediction. Once the tracking process had finished, the results were reconverted to the original scale to be saved. 


\subsection{Worm Model}

At present, there are different skeletonization methods [39,40]. Matlab's bwmorph function was used as a classic skeletonization method to obtain worm skeleton model (color pixels in Figure 2b). The proposed worm model consists of width and color values along the skeleton of each individual, Figure 2c. The width values are obtained using classical skeleton in resulting image after using distance transformation function on segmentation of the image " $k$ ". Grayscale image is shown in Figure 2a, while color values of pixels are obtained using the classical skeleton in gray image in Figure $2 \mathrm{~b}$. The length value is the total number of pixels in the skeleton. The length model is averaged while the C. elegans are separate and tracking progresses.
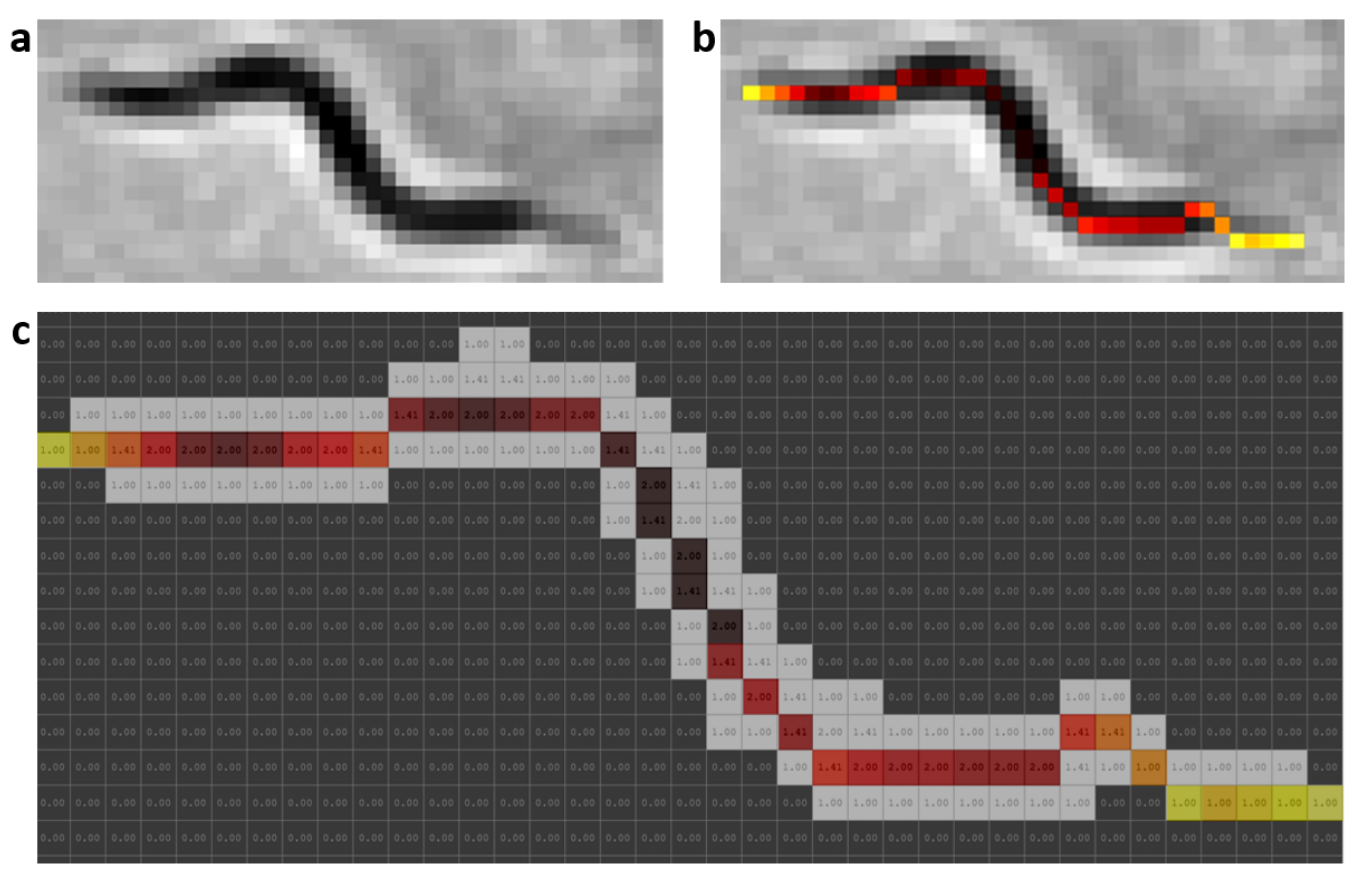

Figure 2. Prediction model. Skeleton gray values were changed with a HOT color map for better visualization. (a) Grayscale image. (b) Color values obtained from grayscale image. (c) Values of widths marked with the colors of the model; the length is the total pixels in the skeleton.

\subsection{Extraction of Possible Solutions}

The skeletonization method proposed in the previous work [35], unlike classical methods, enables the separation of aggregated worms (Figure 3a), creating new paths in the skeleton, and some possible solutions for each worm (Figure $3 \mathrm{~b}-\mathrm{e}$ ). Maximum and minimum values of the width vector are used in the distance to transform images of each segmentation to find this new skeleton as mentioned in [35]. The possible solution skeletons are obtained from a recursive function, which runs through ends and branch points of the new skeleton that overlap the previous segmentation of the worm's body (red circle in Figure 3b-e). 

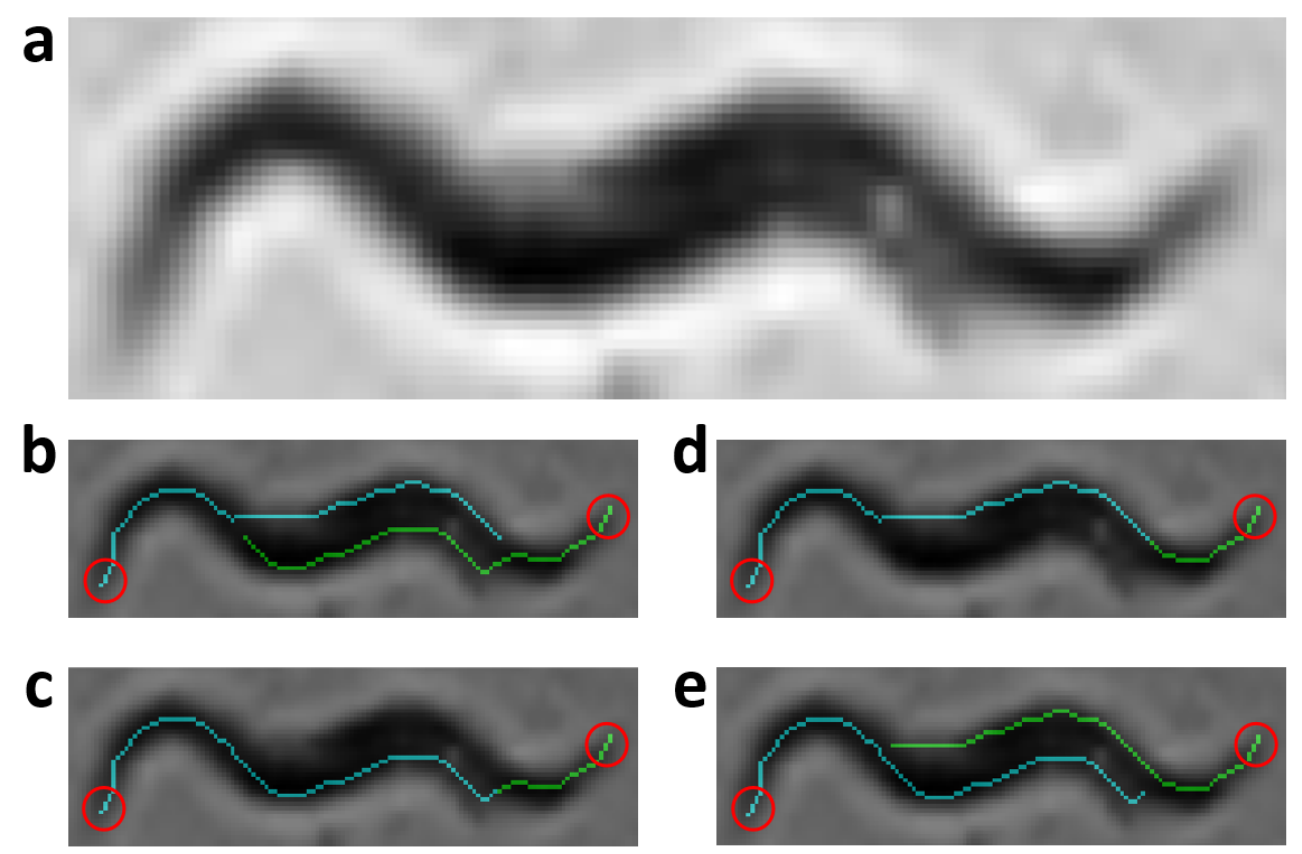

Figure 3. Possible solutions. Circles in red mark the starting point to find the possible skeleton. Cyan and green lines are the possible solutions for each worm. (a) Grayscale image. (b-e) Possible solutions.

\subsection{Optimization Method}

The prediction "S" of the following postures will be the possible solution with the minimum value within all the possible " $P$ " combinations of skeletons in one segmentation (1). The value of each possible combination " $C_{P}$ " of skeletons is obtained from the sum of criteria " $m$ ", for the number of worms " $n$ " in an aggregation (2). The criterion " $C_{p}$ " is evaluated for each possible worm " $\mathrm{i}$ " and for each criterion " $\mathrm{j}$ ". The criteria analyzed were the length of skeleton, overlap with the previous body, completeness, smoothness of the skeleton, noise in segmentation and the colors of each worm.

$$
\begin{gathered}
S=\underset{p}{\arg \min }\left(C_{p}\right) \\
C_{p}=\sum_{j=1}^{m}\left(\sum_{i=1}^{n} C_{j}^{i}\right)
\end{gathered}
$$

The length and color criteria prevent the prediction differing from the model in length and color. The smoothness criterion prevents sudden changes in the direction of the skeletons. The completeness criterion prevents the current segmentation from being incomplete. The overlap criterion with the previous body prevents the identity change during aggregation. Furthermore, the noise criterion prevents the skeleton prediction falling on the plate noise.

The reconstruction of the body of each worm was used for the evaluation of the different criteria. This was performed by using the skeleton pixels in each possible prediction with the width and color values obtained in the model (prediction start), respectively, for each individual.

\subsubsection{Length Criterion}

The length criterion " $C_{L}$ " is obtained from the sum of the multiplication of average squared width $\left[\overline{W_{i}^{2}}\right]$ with the difference in length $\left(\Delta_{i}\right),(3)$. This difference is obtained from subtraction between the model length of each worm $\left(L_{i}\right)$ and length of the skeleton obtained $\left(W L_{i}\right)$. The average squared of the width was used so that the resulting length criterion is as significant as the rest of the criteria where the error in pixels was evaluated. 
Figure 4a shows the aggregation of two worms in a gray scale, and Figure $4 \mathrm{~b}, \mathrm{c}$ shows possible posture predictions where length errors occur.

$$
C_{L}=\sum_{i=1}^{n}\left(\Delta_{i} \overline{W_{i}^{2}}\right)
$$

a

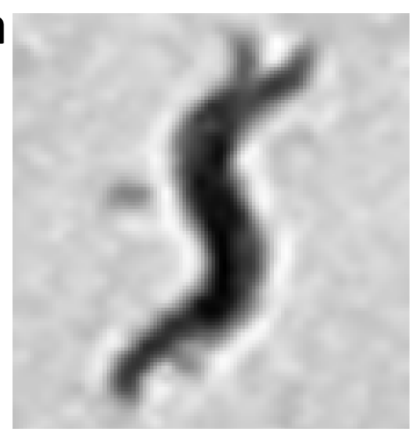

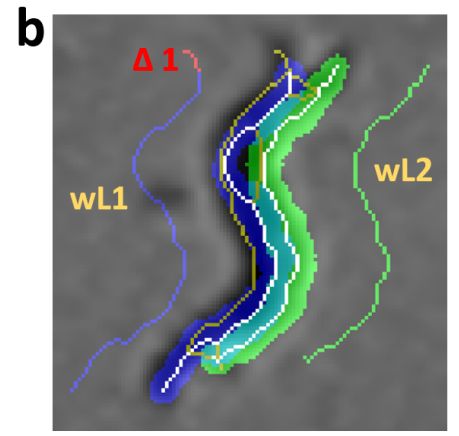

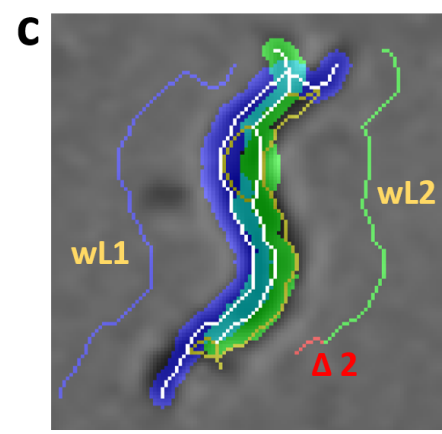

Figure 4. Length criterion evaluation. The pixels in red are the length error. The yellow and white pixels are the resulting skeleton using the improved form of skeletonizing. The white pixels are the pixels of the skeleton prediction, which are used to reconstruct the body of each worm (segmentation in blue and green). (a) Grayscale image. (b) Length criterion evaluation with length error in worm1 (blue). (c) Length criterion evaluation with length error in worm2 (green).

\subsubsection{Overlap Criterion}

The overlap criterion " $C_{O}$ " is obtained from the sum of the absolute difference of the reconstruction of the worm's body in the previous state, $B_{P p x}$, (white dashed line in Figure $5 b, c, e, f$ ) and the current state, $B_{C p x}$, (green and blue segmentation in Figure $5 b, c, e, f$ ) for each possible worm " $i$ " in the aggregation (4) and (5). This is done for all " $\mathrm{m}$ " pixels in each reconstruction. Figure 5a shows in gray scale the aggregation of two worms, Figure $5 \mathrm{~d}$,g shows the next postures predictions (blue and green segmentation), parallel to these in dashed lines showing the previous state $\left(B_{P p x}\right)$ and the rest show the current state $\left(B_{C p x}\right)$. In Figure $5 \mathrm{~d}$ it can be seen that the overlap criterion is low because it belongs to the same worms, while in Figure $5 \mathrm{~g}$ it is higher due to the identity change.

$$
\begin{aligned}
& C_{O}^{i}=\sum_{p x=1}^{m}\left\{\begin{array}{l}
1 \text { if }\left|B_{P p x}-B_{C p x}\right|==1 \\
0
\end{array}\right. \\
& C_{O}=\sum_{i=1}^{n} C_{O}^{i}
\end{aligned}
$$

a

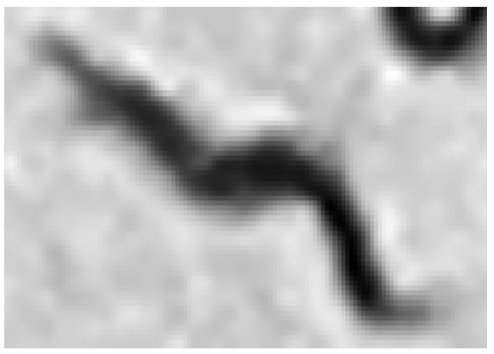

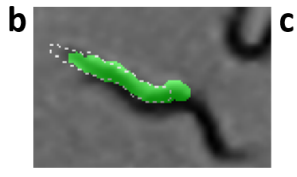
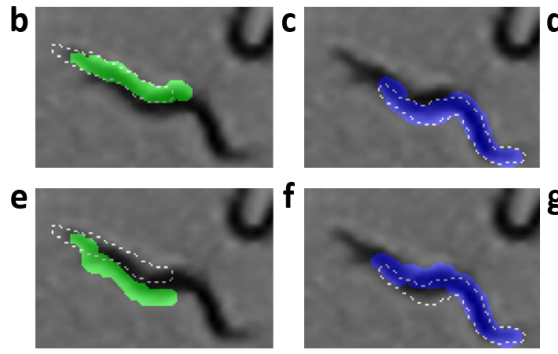

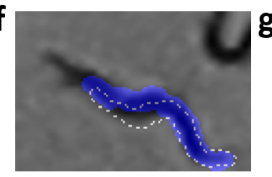

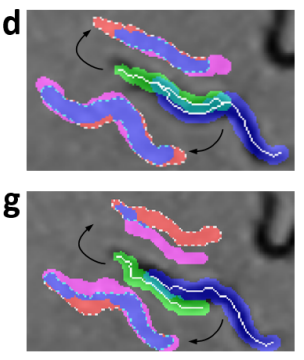

Figure 5. Overlap criterion evaluation. The pixels in red and magenta are the error of overlap with the previous state. The white pixels are the pixels of the skeleton prediction, which are used to reconstruct the body of each worm (segmentation in blue and green). The dashed line segmentation is the previous state segmentation. (a) Grayscale image. (b,e) Previous state $\left(B p s_{p x}\right)$ in white dashed line and current state $\left(B c s_{p x}\right)$ in green. $(\mathbf{c}, \mathbf{f})$ Previous state $\left(B p s_{p x}\right.$ in white dashed line and current state $\left(B c s_{p x}\right)$ in blue. (d) Evaluation with low overlap criterion. (g) Evaluation with high overlap criterion. 


\subsubsection{Completeness Criterion}

The completeness criterion " $C_{C p}$ " is obtained from the sum of the absolute difference of the current segmentation of the image $\left(A_{S}\right)$ and the reconstruction of each possible worm body " $i$ " in the current state $\left(B_{C p x}\right)(6)$ and $(7)$. This is done for all " $m$ " pixels in each reconstruction or current segmentation. Figure 6a shows the aggregation of two worms in gray scale, Figure $6 \mathrm{~b}-\mathrm{d}$ shows completeness values for each image. Figure $6 \mathrm{~b}$ is the correct prediction, while Figure $6 \mathrm{c}$ shows incorrect prediction, due to the identity change and final extremes. Figure $6 \mathrm{~d}$ shows low completeness criterion and an incorrect prediction too, due to the change of identity in both worms.

$$
\begin{gathered}
C_{C p}^{i}=\sum_{p x=1}^{m}\left\{\begin{array}{l}
1 \text { if }\left|A_{S p x}-B_{C p x}\right|==1 \\
0
\end{array}\right. \\
C_{C p}=\sum_{i=1}^{n} C_{C p}^{i}
\end{gathered}
$$

a

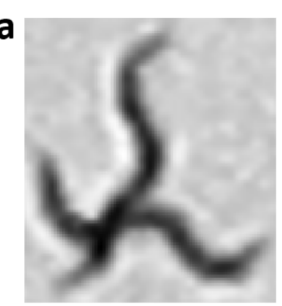

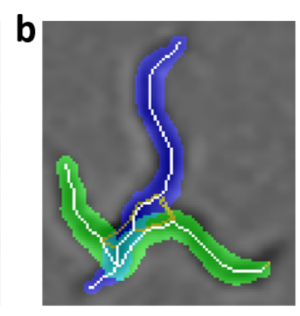
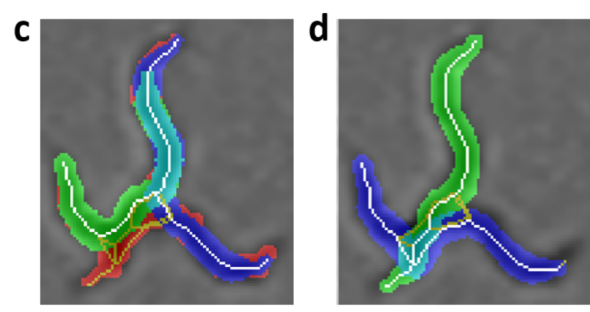

Figure 6. Completeness criterion evaluation. The pixels in red are the completeness error. The yellow and white pixels are the resulting skeleton using the improved form of skeletonizing. The white pixels are the pixels of the skeleton prediction, which are used to reconstruct the body of each worm (segmentation in blue and green). (a) Grayscale image. (b) Correct prediction with low completeness criterion. (c) Incorrect prediction with high completeness criterion. (d) Identities changed and with the same completeness criterion as image $(\mathbf{b})$.

\subsubsection{Smoothness Criterion}

The smoothness criterion " $C_{S}$ " is obtained from the average of the absolute values of the angles obtained for the "nk" pixels of the skeleton and for each worm " $\mathrm{i}$ " in the segmentation (9). For each pixel of the skeleton, there is an angle $\left(\theta_{p x}\right)$, which is obtained by an average of the sum of " $\mathrm{A}$ " angles before and " $\mathrm{n} A$ " angles after divided by the total pixels found " $c$ " (8). The value of " $c$ " will be $2^{*} n A$ if there are "nA" pixels before and after the pixel to be evaluated (8). Figure 7a shows the aggregation of two worms in gray scale, Figure $7 \mathrm{~b}$ shows a possible prediction of skeletons with low softness criterion, while Figure $7 \mathrm{c}, \mathrm{d}$ show possible predictions of skeletons with high softness criterion.

$$
\begin{gathered}
\theta_{p x}=\frac{\sum_{a=x-n A}^{x+n A} \theta_{a}}{c} \\
C_{S}=\sum_{i=1}^{n}\left(\sum_{p x=1}^{n k} \frac{\left|\theta_{p x}\right|}{n k}\right)
\end{gathered}
$$



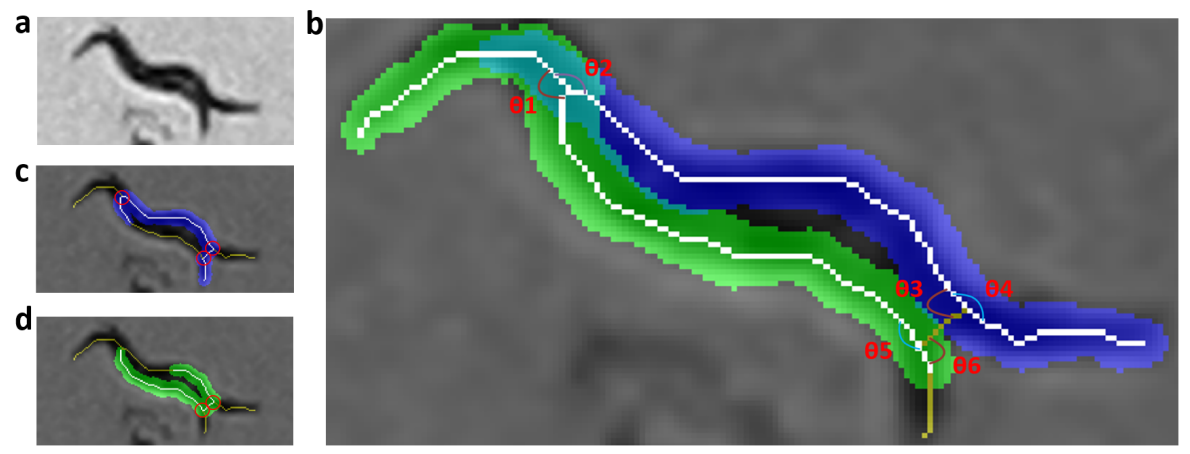

Figure 7. Smoothness criterion evaluation. The yellow and white pixels are the resulting skeleton using the improved skeletonizing method. The white pixels are the pixels of the skeleton prediction, which are used to reconstruct the body of each worm (segmentation in blue and green). (a) Grayscale image. (b) Evaluation of smoothness, angles in red are those that have more weight and increase the index of smoothness. (c) Incorrect prediction of worm1 with high softness criterion. (d) Incorrect prediction of worm2 with high softness criterion.

\subsubsection{Noise Criterion}

The noise criterion " $C_{N}$ " is obtained from the intersection of the noise segmentation $\left(N_{S}\right)$ and the reconstruction of each body of a possible worm " $\mathrm{i}$ " in the current state $\left(B_{C p x}\right)$ (10) and (11). This is done for all " $\mathrm{m}$ " pixels in each reconstruction or noise segmentation. Figure 8 a shows the aggregation of one worm with noise in a gray scale, while Figure $8 b$ shows the correct prediction (segmentation in blue) with a low noise criterion (segmentation in magenta). Figure $8 \mathrm{c}$ shows an incorrect prediction (blue-magenta segmentation) with a high noise index (magenta segmentation).

$$
\begin{aligned}
& C_{N}^{i}=\sum_{p x=1}^{m}\left\{\begin{array}{l}
1 \text { if }\left|N_{S p x} \& B_{C p x}\right|==1 \\
0
\end{array}\right. \\
& C_{N}=\sum_{i=1}^{n} C_{N}^{i}
\end{aligned}
$$

a

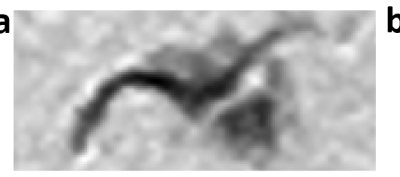

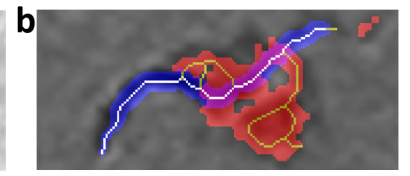

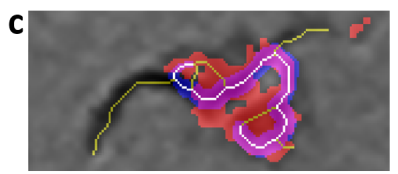

Figure 8. Noise criterion evaluation. The pixels in magenta (intersection of blue and red) are the noise error. The yellow and white pixels are the resulting skeleton using the improved form of skeletonizing. The white pixels are the pixels of the skeleton prediction, which are used to reconstruct the worm's body (segmentation in blue). (a) Grayscale image. (b) Correct prediction with low noise criterion. (c) Incorrect prediction with high noise criterion.

\subsubsection{Color Criterion}

The color criterion " $\mathrm{C}_{\mathrm{Cl}}$ " is obtained by adding all the pixels with an absolute difference greater than the threshold value $(\mathrm{U}=1)$ of the color model $\left(C_{M}\right)$ with respect to the reconstruction of the current body $\left(C_{A}\right)(12)$ for each worm in the aggregation (13). This means that the error only increases the value by 1 if the absolute difference between a pixel of the reconstruction of the body of the possible worm " $\mathrm{i}$ " using the color model $\left(C_{M p x}\right)$ and the reconstruction of the same worm " $\mathrm{i}$ " in the current segmentation $\left(C_{A p x}\right)$ is greater than the threshold value. This is done for all " $\mathrm{m}$ " pixels in the reconstruction. For both worm body reconstructions (model and current prediction), the same width and length values are used, in order to compare pixels 1 to 1 . Figure 9a shows the aggregation of two 
worms in gray scale, while Figure $9 b, c$ shows the comparison of each prediction with its respective model.

$$
\begin{aligned}
& C_{C l}^{i}=\sum_{p x=1}^{m}\left\{\begin{array}{l}
1 \text { if }\left|C_{M p x}-C_{A p x}\right|>U \\
0
\end{array}\right. \\
& C_{C l}=\sum_{i=1}^{n} C_{C l}^{i}
\end{aligned}
$$
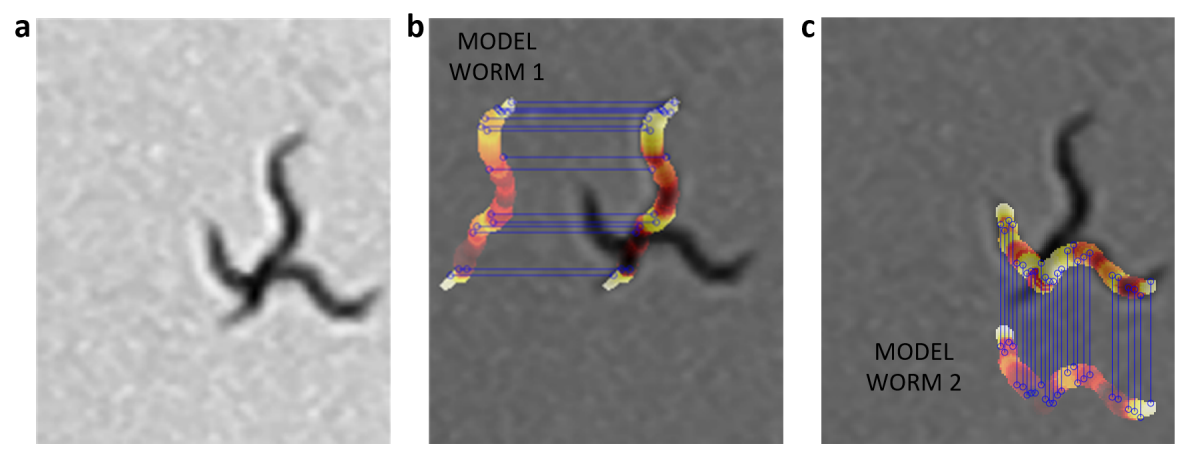

Figure 9. Color criterion evaluation. For each possible skeleton, its color values are obtained using the current grayscale image, and with those color values and the width values of the model, each worm is reconstructed and compared with the reconstruction of the model (model worm). The gray values were changed for a HOT color map in order to better visualize them. The blue lines indicate those pixels different from the model. (a) Grayscale image. (b) Comparison of worm1 model with current prediction of worm1. (c) Comparison of worm2 model with current prediction of worm2.

\subsection{Evaluation Method}

Manually labeled skeletons were used as a reference to compare all the results. These skeletons were obtained using an application designed to select each pixel belonging to the skeleton of each worm one by one in the image sequence. This operation was performed for all 3779 postures of the 70 plates used. The shape of these nematodes was recovered using a disk-shaped dilation operation of radius equal to half the width (approx. 2 pixels) on the skeletons obtained.

The Jaccard coefficient, or intersection over the union (IoU), was used to measure the degree of precision in locating worms (14). As its name indicates, it is obtained by dividing the total area of the intersection by the union of the elements [41]. For the evaluation, the area of the reconstructed bodies of the manually labeled skeletons was used, skeletons using the skeletonization method proposed in [35] and the classical skeletonization method using the Matlab bwmorph command.

$$
I o U=\frac{\sum P_{w 1} \cap P_{w 2}}{\sum P_{w 1} \bigcup P_{w 2}}
$$

The IoU index was expected to be higher because a predicted pose (Figure 10b) is compared to an annotated ground-true pose (Figure 10a), which must overlap (Figure 10c-e). The results for the example below are IoU $=0.9784,0.5667$, and 0.2649 , respectively.

Matlab 2018b Machine Learning Toolbox was used to obtain the comparison statistics between prediction models (Appendix A Figures A1a,b-A4a,b) and the two skeletonization methods (Appendix A Figures A5a,b and A6a,b). The Kolmogorov-Smirnov normality test was used for large samples $(n>50)$ and the Wilcoxon Signed Ranks test. 
a

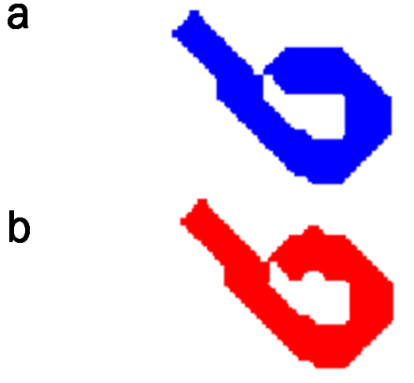

d

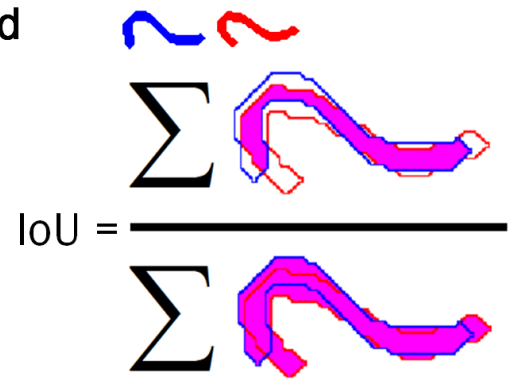

C

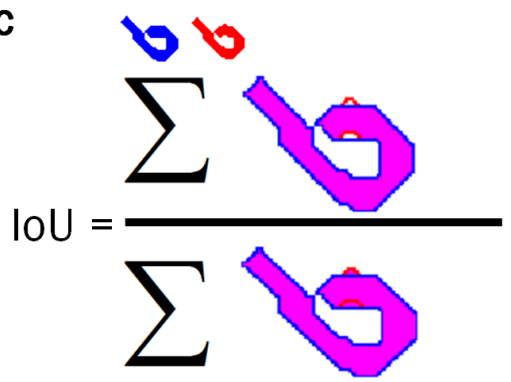

e

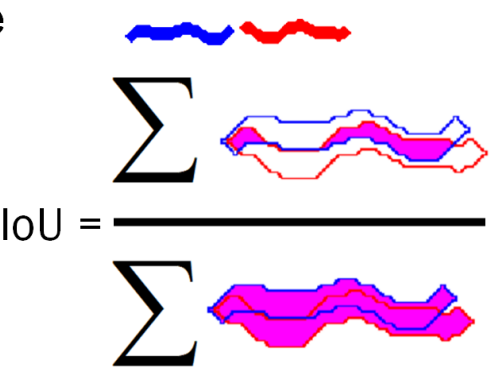

Figure 10. IoU index. This index evaluates how close the response of the automatic method comes to the reference, and compares both results to determine the improvement of one method over the other. The higher the IoU value, the closer the response comes to the reference. The evaluation is performed by reconstructing the skeletons obtained with a radio 2 disk. (a) Reconstructed body of the manually labeled skeleton. (b) Reconstructed body of the skeleton obtained using the new skeletonization method [35] or the classical method. (c-e) Evaluation of the reconstructed skeletons.

\section{Results}

Experiments were performed with 70 plates. Of these, 54 corresponded to plates with 10 and 15 worms, one plate with 30 worms, four plates with 60 worms, and 11 plates with 100 worms, totaling 65,400 worm poses. All these data were analyzed to obtain contact between worms and noise. As demonstrated in [5,14], a higher number of worms per plate will increase the likelihood of contact between them. Nematodes studied were young-adult wild-type (N2) and CB1370, daf-2 (e1370), as mentioned above.

53 tracks with 3240 poses were used to evaluate aggregation between worms, and 17 tracks with 509 poses for aggregation between worms and noise. The IoU index was used to evaluate the percentage of success in tracking the worms and also to compare both skeletonization methods. The area of worm bodies reconstructed from skeletons obtained manually and skeletons obtained with the two skeletonization methods (new and classical) was used to evaluate the IoU index.

Different prediction models were implemented in order to find the most significant criteria. The name of each model has been coded using letters from the criteria names. " $\mathrm{O}$ " for overlap, " $\mathrm{L}$ " for length, "Cp" for completeness, "N" for noise, " $\mathrm{S}$ " for smoothness,

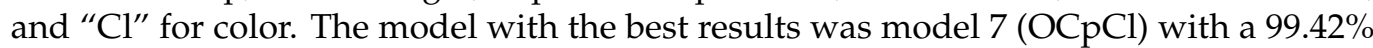
percentage accuracy in aggregated worm tracks and an IoU value of 0.70 in average. Figure 11a-e, shows an example using the model 7 , in this image you can see the evaluation of the three criteria of this model and optimization result. Some examples of aggregation cases are presented at the end of the Appendix B using model 7 (Figures A7-A11). 

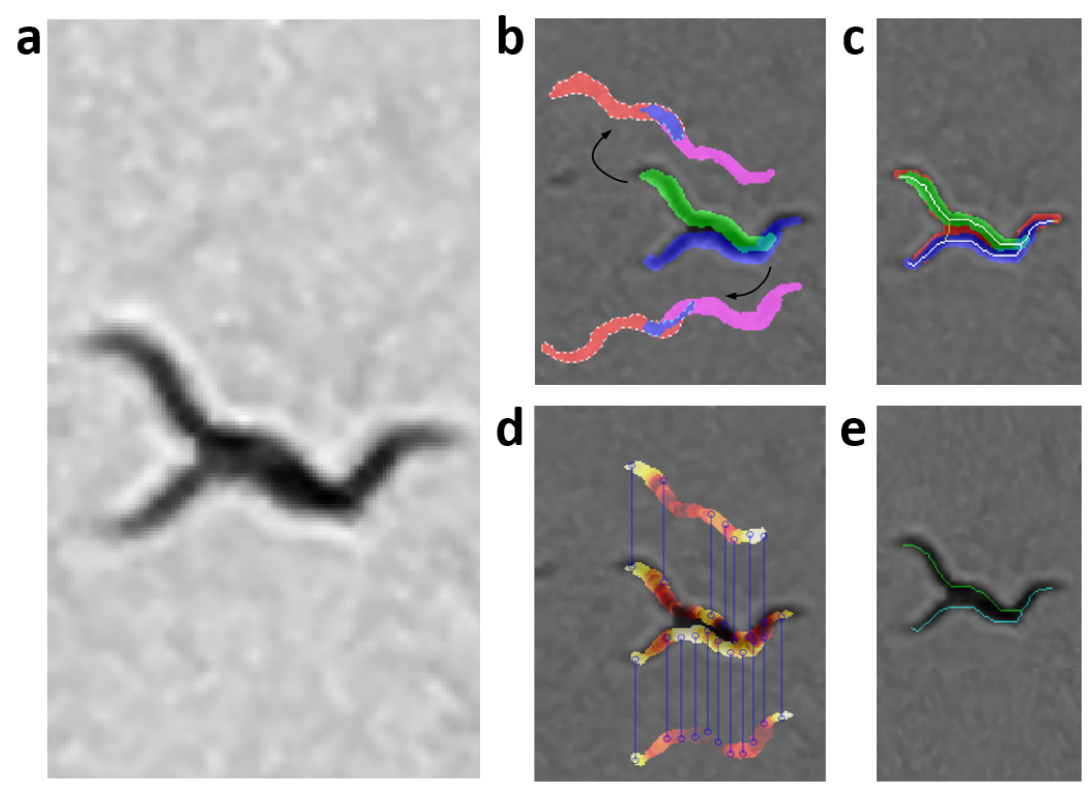

Figure 11. Model 7 evaluation. The yellow and white pixels are the resulting skeleton using the improved form of skeletonizing. The white pixels are the pixels of the skeleton prediction, which are used to reconstruct the body of each worm (segmentation in blue and green). (a) Grayscale image. (b) Overlap criterion evaluation. (c) Completeness criterion evaluation. (d) Color criterion evaluation. (e) Optimization result.

To measure the percentage accuracy in tracking the worms, non-zero IoU values were used, from the beginning to the end of the tracks. The accuracy of the results of the different prediction models using the 2 skeletonization methods are shown in Table 3. In addition, the average IoU value was obtained for all the prediction models (see Table 4), from the beginning of the aggregation to the end. Then, 790 poses were used in aggregations between worms and 509 poses for worms aggregated with noise, 1299 poses in total.

Table 3. Comparative table of percentage accuracy of postures for models and methods. The table shows the percentage accuracy of poses (skeletons) for each model and method used during the tracking of C. elegans. Overall, 3240 poses were used to evaluate tracks where there is aggregation of two or more worms, and 509 poses to evaluate the aggregation between worms and noise on the plate.

\begin{tabular}{clcc}
\hline \multicolumn{4}{c}{ Worms Aggregation } \\
\hline $\mathbf{N}^{\circ}$ & Model & New & Classical \\
\hline 1 & O & 98.49 & 97.80 \\
2 & OL & 98.15 & 97.99 \\
3 & OCp & 99.13 & 98.09 \\
4 & ON & 98.09 & 97.88 \\
5 & OS & 98.07 & 97.72 \\
6 & OCl & 98.57 & 98.20 \\
7 & OCpCl & $\mathbf{9 9 . 4 2}$ & $\mathbf{9 8 . 7 3}$ \\
\hline
\end{tabular}


Table 4. Summary of model and method comparison. This table shows the results for each prediction model and two skeletonization methods, new [35] and classical (using the Matlab bwmorph function). The results column indicates the percentage value of the improvement using the new skeleton with respect to the classic skeletonization.

\begin{tabular}{|c|c|c|c|c|c|c|c|}
\hline & & & \multicolumn{2}{|c|}{ Average IoU } & \multicolumn{2}{|c|}{ Standard Deviation } & Results \% \\
\hline $\mathbf{N}^{\circ}$ & Model & Total Pose & New & Classical & New & Classical & Improvement \\
\hline 1 & $\mathrm{O}$ & 1299 & 0.64 & 0.63 & 0.23 & 0.22 & 0.39 \\
\hline 2 & OL & 1299 & 0.63 & 0.63 & 0.23 & 0.22 & 0.10 \\
\hline 3 & OCp & 1299 & 0.69 & 0.67 & 0.19 & 0.19 & 2.61 \\
\hline 4 & ON & 1299 & 0.63 & 0.63 & 0.24 & 0.22 & 0.50 \\
\hline 5 & OS & 1299 & 0.63 & 0.63 & 0.23 & 0.22 & 0.04 \\
\hline 6 & $\mathrm{OCl}$ & 1299 & 0.65 & 0.65 & 0.23 & 0.20 & -0.15 \\
\hline 7 & $\mathrm{OCpCl}$ & 1299 & 0.70 & 0.67 & 0.19 & 0.18 & 2.66 \\
\hline
\end{tabular}

\section{Comparison with Other Trackers}

At present, automatic or semi-automatic trackers discard the data of tracks where there are overlap or body contacts, due to the difficulty of solving the identity of each individual in these situations. Our method does solve these situations, so a direct comparison cannot be made with the results of other trackers.

To compare our method graphically with other trackers (tierpsy-tracker [22], WFNTP.v3 [23]), labeled data was first shaded in different grays and overlapped predicted data in colors (Figure 12a-c). Errors for each comparison are shown in grayscale. The comparison was made with bodies reconstructed from skeletons (skeleton dilation with disk equal to 2), except for WF-NTP.v3 [23], whose results are skeleton centroid points. The tierpsy-tracker [22] multi-tracker, Figure 12a, did not resolve path 2 where aggregation occurs, while the other tracks $(1,3,4)$ were partially resolved, due to the occlusions that the worms make on themselves. The multi-tacker WF-NTP.v3 [23], Figure 12b, did not solve the identity problem in track 2 . Furthermore, like the previous multi-tracker it presented problems in the tracks with occlusions $(1,3,4)$. Model 7 , on the other hand, had almost zero errors, tracked all worms, resolved the identity in the aggregation of track 2 and the occlusions in the remaining tracks, Figure 12c.
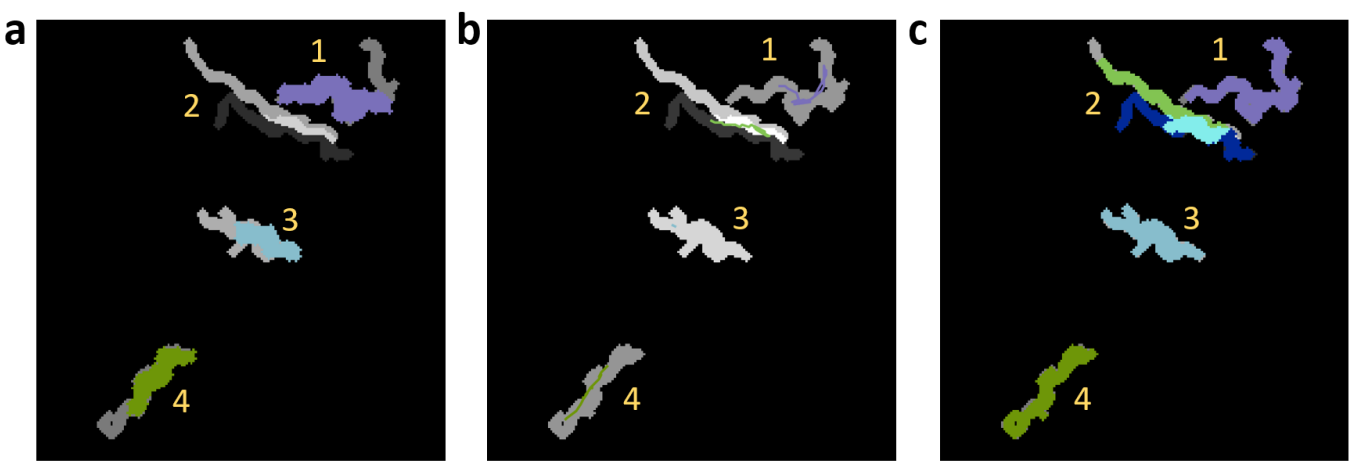

Figure 12. Comparison of trackers. Comparison of reconstruction of C. elegans bodies between labeled data (shaded in grays) and predictions obtained with different trackers (shaded in colors). (a) Results obtained using tierpsy-tracker [22]. (b) Labeled data (grays) compared with colored lines that connect centroids obtained using WF-NTP.v3 [23]. (c) Results obtained using model 7.

\section{Discussion}

Caenorhabditis elegans are aggregated in different ways, such as aggregation of end parts (head or tail), partial aggregation of bodies, and aggregation of parallel bodies, among others. The experiments were conducted with the above mentioned methods with a single criterion and found that the overlapping criterion with the previous prediction is the most 
significant. This criterion allows part of the previous state to be preserved, helping to solve the next state. A result of $98.49 \%$ was obtained using this criterion individually. However, when aggregation gives rise to an overlap in most worms, it is difficult to identify them, even for human observers. To solve this problem, different tests were designed, combining the criteria mentioned in the optimization methods section.

The completeness in cases of aggregation, where there are overlaps between aggregated bodies, partial and total identity changes could be obtained, as observed in the optimization method Figure $6 \mathrm{c}, \mathrm{d}$. When using the overlap criterion with completeness criterion, a percentage accuracy of $99.13 \%$ was achieved. As shown in Appendix A (Figures A1a,b and A2a,b) this criterion is statistically significant, helping to solve some cases where the overlap criterion presented problems, Figure $5 \mathrm{~b}$.

The color criterion also helped to improve worm prediction, although not so significant (Appendix A Figures A3a,b and A4a,b). The end portion of C. elegan (tail) has a greater number of light pixels (higher gray levels) than the head, where there are fewer. These features are important because before, during and after an aggregation one of the final parts remains visible, and with this color singularity a more accurate prediction can be obtained, helping to solve the identity problem after an aggregation between worms or noise. The use of these three criteria (overlap, completeness, and color) allowed us to obtain a percentage accuracy of $99.42 \%$.

The length, smoothness, and noise criteria were discarded because when they were used individually or together with the overlap criterion, their percentage accuracy decreased. On the one hand, the problem with the smoothness criterion was due to the low resolution of the worms in our images, which provided a poor estimate of this criterion. On the other hand, the problem with the length criterion was due to errors in the length model and the increase or decrease in the length of worms owing to overlaps. The problem with the noise criterion was due to, when the worm is visualized against background noise, many possible solutions were generated.

Finally, it is worth mentioning that the best combination of criteria depends on image quality. In this work, we demonstrated that the combination of the three criteria mentioned above (overlap, completeness, and color) was the best option for automatic tracking of interaction behaviors among C. elegans (contacts or overlapping) with our low-resolution dataset.

\section{Conclusions}

This paper presents a method for tracking multiple C. elegans in standard Petri dishes where some worms can come into contact or overlap. This method was evaluated in a difficult scenario using a low-image resolution and a low frame rate. Using an optimizer with the appropriate criteria (overlap, completeness, and color) was shown to solve many worm overlap and contact situations. The accuracy obtained under these conditions was $99.42 \%$ and $98.73 \%$ using the modified skeleton algorithm and the classical skeleton algorithm, respectively. In addition, the proposed method employs an improved active backlight system and an improved skeletonization algorithm. The active backlight system provides fixed gray levels in all captured images, which allows automatic segmentation using a fixed threshold. The improved skeletonization algorithm uses width information from each worm model to extract skeletons, enabling the tracking of worms moving in parallel (side by side). Our proposal, unlike other trackers that discard worm overlaps and contacts, solves many of these situations, increasing the number of worms tracked in a Petri dish and therefore paving the way to automate new assays where interaction between worms occurs. 
Author Contributions: Conceptualization, P.E.L.C. and A.-J.S.-S.; methodology, P.E.L.C. and A.-J.S.S.; software, P.E.L.C.; validation, P.E.L.C., J.C.P., A.-J.S.-S. and A.G.G.; formal analysis, P.E.L.C. and A.-J.S.-S.; investigation, P.E.L.C. and A.-J.S.-S.; resources, A.-J.S.-S.; data curation, P.E.L.C., J.C.P. and A.G.G.; writing-original draft preparation, P.E.L.C. and A.-J.S.-S.; writing-review and editing, P.E.L.C., J.C.P., A.G.G. and A.-J.S.-S.; visualization, P.E.L.C. and A.-J.S.-S.; supervision, A.-J.S.-S.; project administration, A.-J.S.-S.; funding acquisition, A.-J.S.-S. All authors have read and agreed to the published version of the manuscript.

Funding: This study was supported by the Plan Nacional de I+D with Project RTI2018-094312-B-I00, FPI Predoctoral contract PRE2019-088214 and by European FEDER funds.

Institutional Review Board Statement: Not applicable.

Informed Consent Statement: Not applicable.

Data Availability Statement: The program was developed in Matlab2018b with Windows 10 and works correctly in later versions with Image Processing, Communications and Bioinformatics toolbox. Its source code is in GitHub. It is open-source MIT (Massachusetts Institute of Technology) and can be downloaded from the repository at https://github.com/playanaC/WT_ISA, accessed on 17 August 2021. The dataset with all aggregation experiments can be downloaded from https:/ / activevision.ai2.upv.es/wp-content/uploads/2021/02/dataset_skeletons.zip, accessed on 17 August 2021.

Acknowledgments: ADM Nutrition, Biopolis S.L. and Archer Daniels Midland supplied the C. elegans plates. Some strains were provided by the CGC, which is funded by NIH Office of Research Infrastructure Programs (P40 OD010440). Maria-Gabriela Salazar-Secada developed the skeleton annotation application. Jordi Tortosa-Grau annotated worm skeletons.

Conflicts of Interest: No conflict of interest exists.

\section{Appendix A}

Appendix A.1. IoU Comparison in Models 3 and 1

a

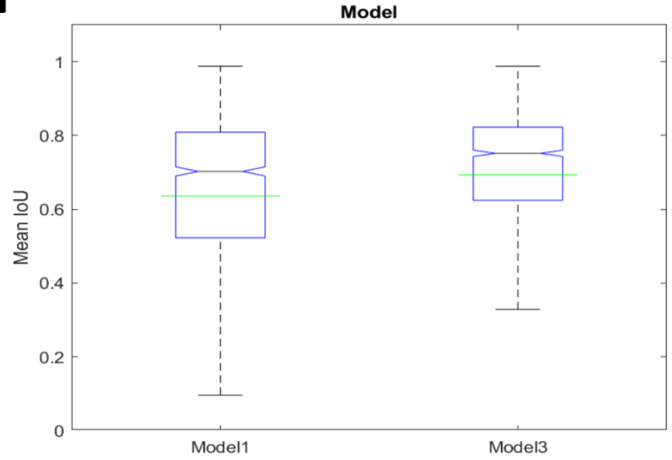

b Kolmogorov Smirnov test

\begin{tabular}{|c|c|}
\hline & Diff \\
\cline { 1 - 1 }$N$ & 1299 \\
\cline { 1 - 1 } Minimun & -0.7073 \\
\cline { 1 - 1 } Maximun & 0.8825 \\
\cline { 1 - 1 } Mean & 0.0575 \\
\cline { 1 - 1 } Std. Deviation & 0.1721 \\
\cline { 1 - 1 } p-value & $8.238 \mathrm{E}-96$ \\
\hline
\end{tabular}

Figure A1. Box plot and normality test of the difference of both models. (a) Box plot, green line indicates the mean in both graphs, and gray line indicates the median. Model1, $\mathrm{N}=1299$, mean $=0.6358$, median $=0.7027$, std. deviation $=0.2289$, variance $=0.0540$. Model3, $\mathrm{N}=1299$, mean $=0.6933$, median $=0.7517$, std. deviation $=0.1940$, variance $=0.0376$. $(\mathbf{b})$ Normality test on the difference of methods (Model 3-Model 1). The $p$-value obtained was 8.24E-96 less than the significance value of 0.05, so the null hypothesis was rejected and the alternative hypothesis $\mathrm{H} 1$ was accepted (data did not come from normal distribution). Once the alternative hypothesis was accepted, Wilcoxon signed ranks test was used to evaluate both methods. 


\section{a Wilcoxon Signed Ranks Test b}

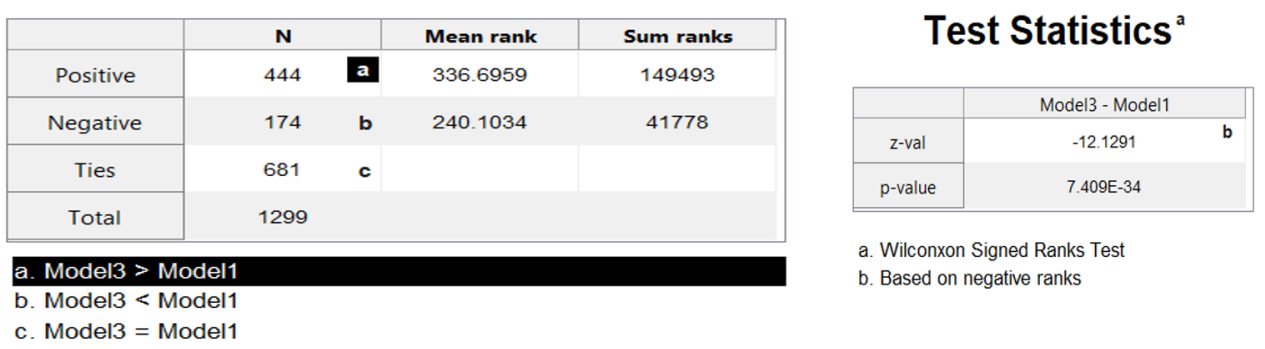

Figure A2. Wilcoxon signed rank test. (a) The Wilcoxon signed rank test table shows the difference that exists in 2 related samples through positive, negative and tie ranges. (b) $p$-value obtained with Wilcoxon rank test was 741E-34 less than the significance value of 0.05 , so it was concluded that there was a statistically significant difference between both models.

Appendix A.2. IoU Comparison in Models 6 and 1

a

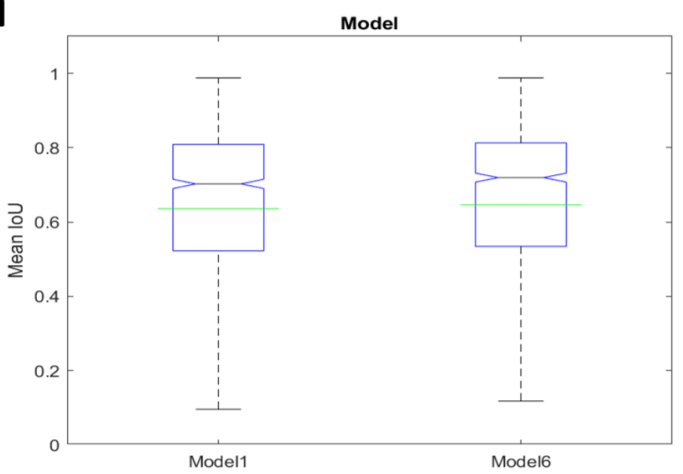

b Kolmogorov Smirnov test

\begin{tabular}{|c|c|}
\hline & Diff \\
\cline { 1 - 1 } $\mathrm{N}$ & 1299 \\
\cline { 1 - 1 } Minimun & -0.8326 \\
\cline { 1 - 1 } Maximun & 0.7590 \\
\cline { 1 - 1 } Mean & 0.0106 \\
\cline { 1 - 1 } Std. Deviation & 0.1476 \\
\cline { 1 - 1 } p-value & $1.477 \mathrm{E}-151$ \\
\hline
\end{tabular}

Figure A3. Box plot and normality test of the difference of both models. (a) Box plot, green line indicates the mean in both graphs, and gray line indicates the median.Model1, $\mathrm{N}=1299$, mean $=0.6358$, median $=0.7027$, std. deviation $=0.2289$, variance $=0.0540$. Model6, $\mathrm{N}=1299$, mean $=0.6464$, median $=0.7197$, std. deviation $=0.2300$, variance $=0.0529$. (b) Normality test on the difference of methods (Model 6-Model 1). The $p$-value obtained was 1.48E-151 less than the significance value of 0.05 , so the null hypothesis was rejected and the alternative hypothesis $\mathrm{H} 1$ was accepted (data did not come from normal distribution). Once the alternative hypothesis was accepted, Wilcoxon signed ranks test was used to evaluate both methods.

\section{a Wilcoxon Signed Ranks Test b}

\begin{tabular}{|c|cc|c|c|}
\hline & N & Mean rank & Sum ranks \\
\hline Positive & 271 & a & 275.5277 & 74668 \\
\cline { 1 - 2 } Negative & 254 & b & 249.6339 & 63407 \\
\cline { 1 - 1 } Ties & 774 & c & & \\
\cline { 1 - 1 } Total & 1299 & & \\
\hline
\end{tabular}

Test Statistics ${ }^{a}$

\begin{tabular}{|c|c|c|}
\hline & Model6 - Model & \\
\hline$z$-val & -1.6191 & b \\
\hline$p$-value & 0.1054 & \\
\hline
\end{tabular}

a. Wilconxon Signed Ranks Test b. Based on negative ranks

\section{b. Model $6<$ Model 1}

c. Model $6=$ Model 1

Figure A4. Wilcoxon signed rank test. (a) The Wilcoxon signed rank test table shows the difference that exists in two related samples through positive, negative and tie ranges. (b) $p$-value obtained with Wilcoxon rank test was 0.1054 less than the significance value of 0.05 , so it was concluded that there was not a statistically significant difference between both models. 
Appendix A.3. IoU Comparison New and Classical Method (Model 7)

a

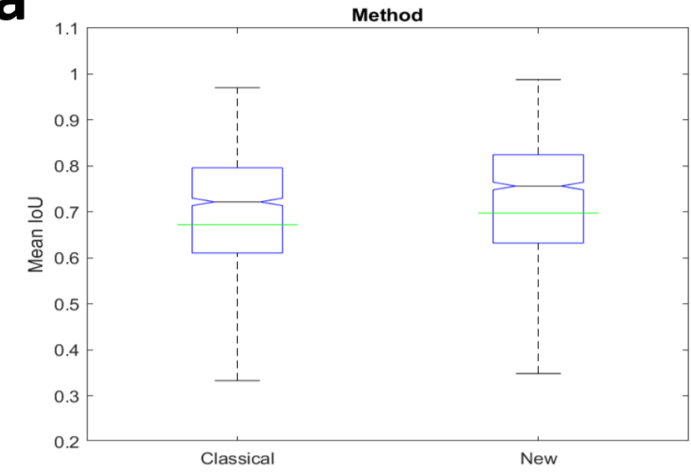

b Kolmogorov Smirnov test

\begin{tabular}{|c|c|}
\hline & Diff \\
\cline { 1 - 1 }$N$ & 1299 \\
\cline { 1 - 1 } Minimun & -0.5879 \\
\cline { 1 - 1 } Maximun & 0.8498 \\
\cline { 1 - 1 } Mean & 0.0256 \\
\cline { 1 - 1 } Std. Deviation & 0.1416 \\
\cline { 1 - 1 } p-value & $1.262 \mathrm{E}-152$ \\
\hline
\end{tabular}

Figure A5. Box plot and normality test of the difference of both methods. (a) Box plot, green line indicates the mean in both graphs, and gray line indicates the median. Classical method, $\mathrm{N}=1299$, mean $=0.6719$, median $=0.7218$, std. deviation $=0.1842$, variance $=0.0339$. New method, $\mathrm{N}=1299$, mean $=0.6975$, median $=0.7562$, std. deviation $=0.1929$, variance $=0.0372$. (b) Normality test on the difference of methods (New-classical). The $p$-value obtained was 1.26E-152 less than the significance value of 0.05 , so the null hypothesis was rejected and the alternative hypothesis $\mathrm{H} 1$ was accepted (data did not come from normal distribution). Once the alternative hypothesis was accepted, Wilcoxon signed ranks test was used to evaluate both methods.

\section{a Wilcoxon Signed Ranks Test b}

\begin{tabular}{|c|c|c|c|c|}
\hline & $\mathbf{N}$ & & Mean rank & Sum ranks \\
\hline Positive & 807 & a & 661.8278 & 534095 \\
\hline Negative & 468 & b & 596.9124 & 279355 \\
\hline Ties & 24 & c & & \\
\hline Total & 1299 & & & \\
\hline
\end{tabular}

Test Statistics ${ }^{a}$

\begin{tabular}{|c|c|}
\hline$z$-val & New -Classical \\
\hline p-value & -9.6858 \\
\hline
\end{tabular}

a. Wilconxon Signed Ranks Test b. Based on negative ranks

Figure A6. Wilcoxon signed rank test. (a) The Wilcoxon signed rank test table shows the difference that exists in two related samples through positive, negative and tie ranges. (b) $p$-value obtained with Wilcoxon rank test was $3.46 \mathrm{E}-22$ less than the significance value of 0.05 , so it was concluded that there was a statistically significant difference between both methods. 


\section{Appendix B}

Appendix B.1. Evaluation Examples with Model 7
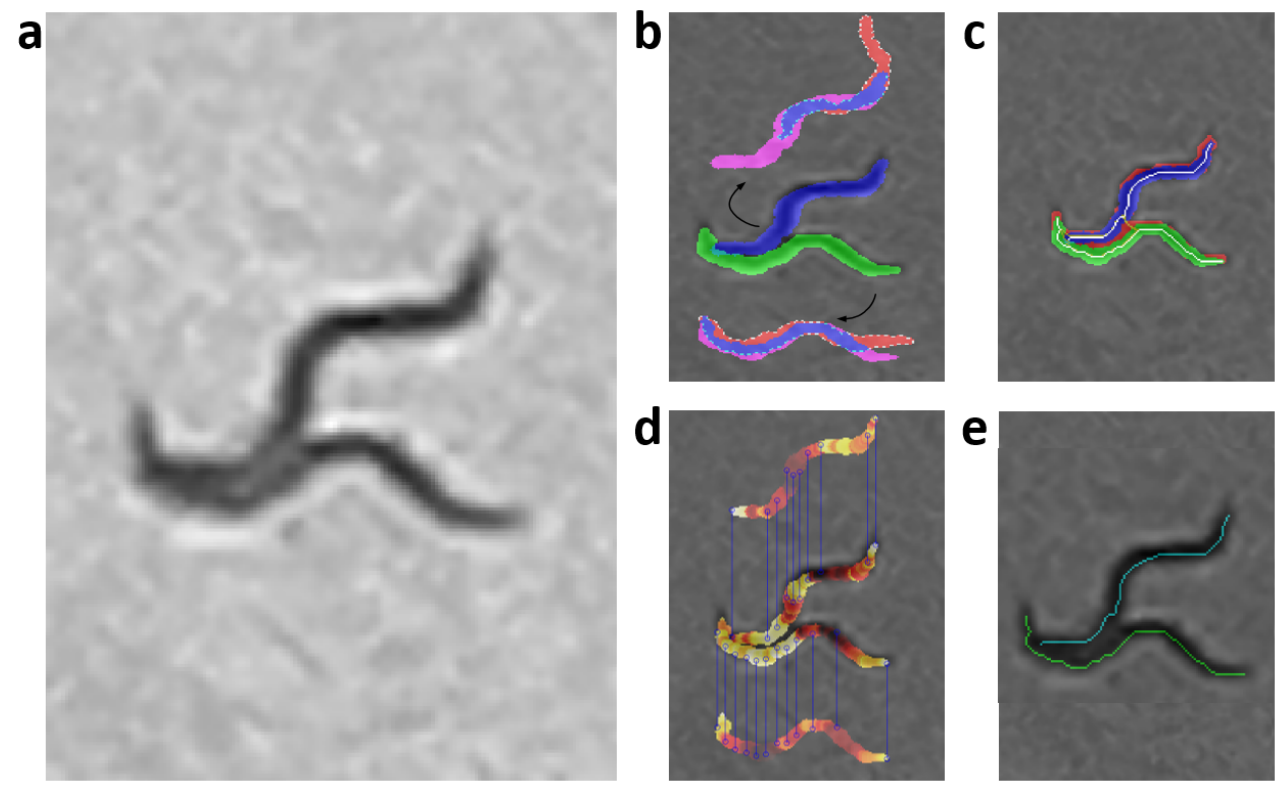

Figure A7. Model 7 evaluation, example 1. The yellow and white pixels are the resulting skeleton using the improved form of skeletonizing. The white pixels are the pixels of the skeleton prediction, which are used to reconstruct the body of each worm (segmentation in blue and green). (a) Grayscale image. (b) Overlap criterion evaluation. (c) Completeness criterion evaluation. (d) Color criterion evaluation. (e) Optimization result.

a

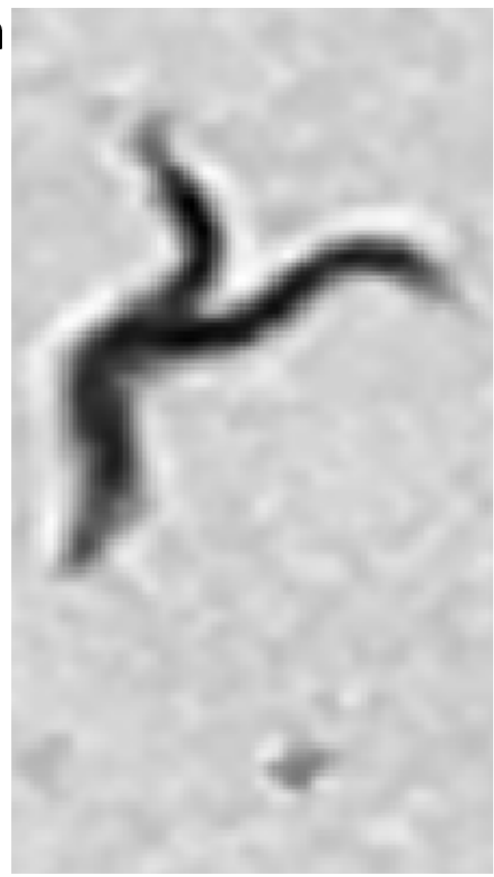

b

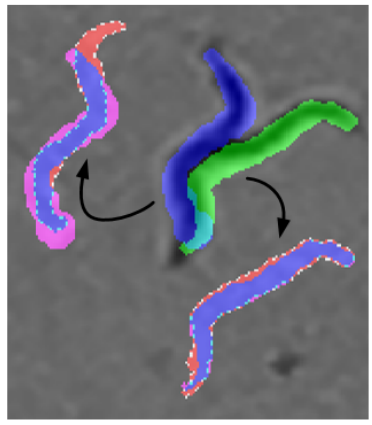

d

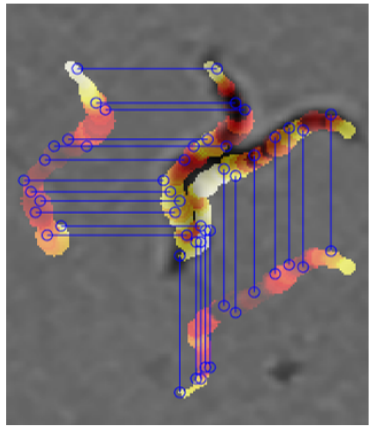

C

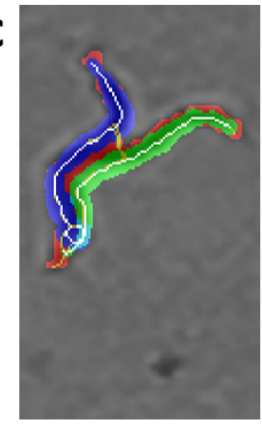

e

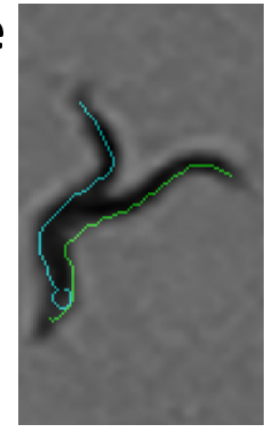

Figure A8. Model 7 evaluation, example 2. The yellow and white pixels are the resulting skeleton using the improved form of skeletonizing. The white pixels are the pixels of the skeleton prediction, which are used to reconstruct the body of each worm (segmentation in blue and green). (a) Grayscale image. (b) Overlap criterion evaluation. (c) Completeness criterion evaluation. (d) Color criterion evaluation. (e) Optimization result. 

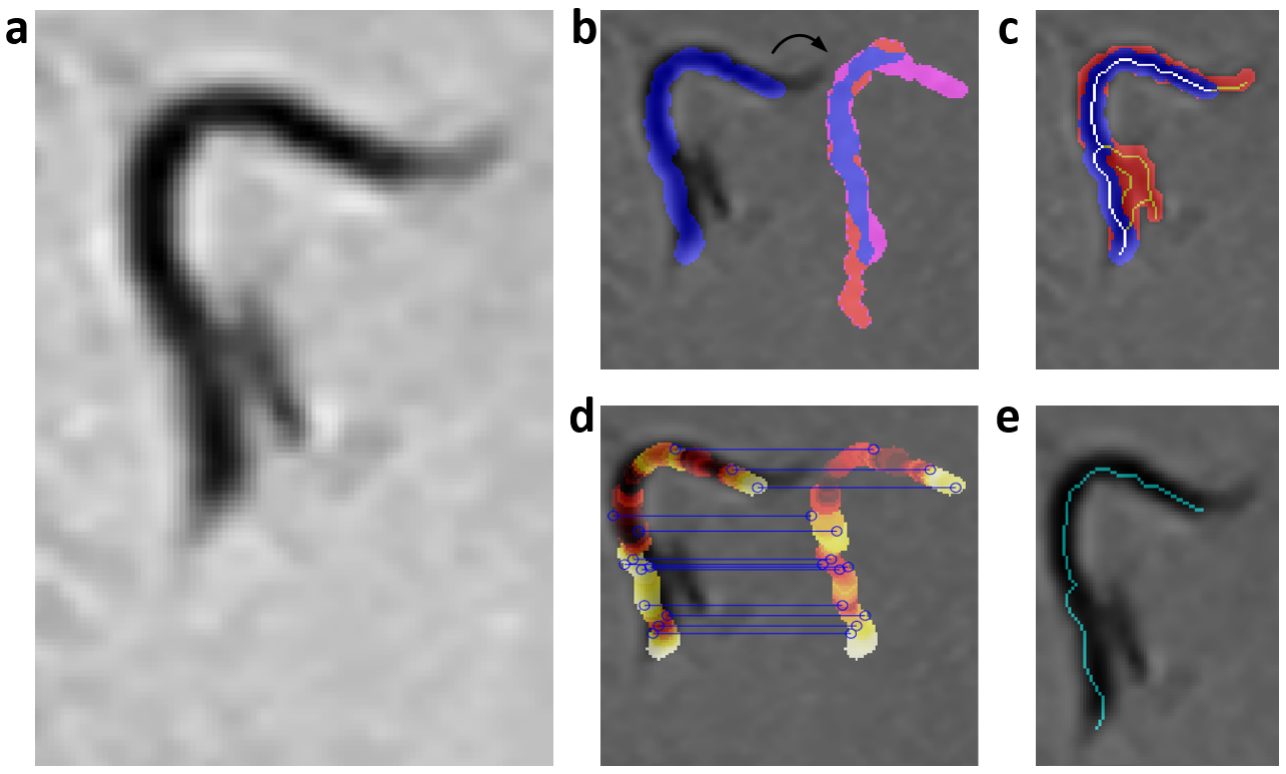

Figure A9. Model 7 evaluation, example 3. The yellow and white pixels are the resulting skeleton using the improved form of skeletonizing. The white pixels are the pixels of the skeleton prediction, which are used to reconstruct the body of each worm (segmentation in blue and green). (a) Grayscale image. (b) Overlap criterion evaluation. (c) Completeness criterion evaluation. (d) Color criterion evaluation. (e) Optimization result.

\section{Appendix B.2. Failure Cases}
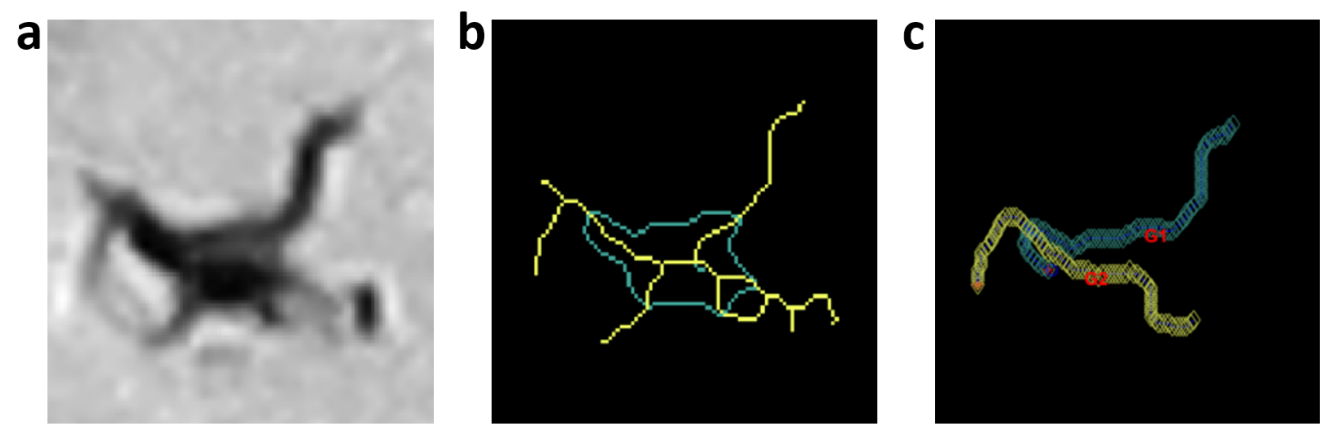

Figure A10. Model 7 evaluation, example 4. Errors occurred by the presence of noise. (a) Grayscale image. (b) Resulting skeleton using the improved form of skeletonizing. (c) Optimization result.
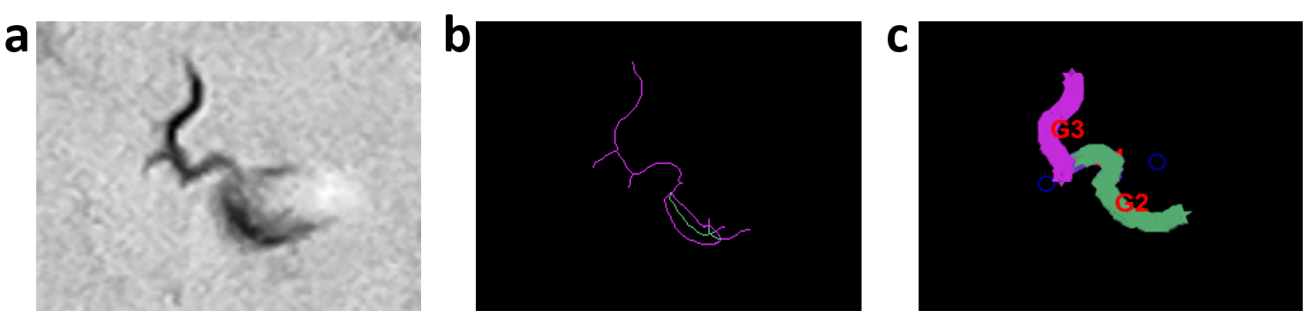

Figure A11. Model 7 evaluation, example 5. Errors occurred by the presence of noise. (a) Grayscale image. (b) Resulting skeleton using the improved form of skeletonizing (c) Optimization result.

\section{References}

1. Olsen, A.; Gill, M.S. Ageing: Lessons from C. elegans; Springer International Publishing: Cham, Switzerland, 2017 ; pp. $2-5$.

2. Teo, E.; Lim, S.J.; Fong, S.; Larbi, A.; Wright, G.D.; Tolwinski, N.; Gruber, J. A high throughput drug screening paradigm using transgenic Caenorhabditis elegans model of Alzheimer's disease. Transl. Med. Aging 2020, 4, 11-21. [CrossRef]

3. Kim, M.; Knoefler, D.; Quarles, E.; Jakob, U.; Bazopoulou, D. Automated phenotyping and lifespan assessment of a C. elegans model of Parkinson's disease. Transl. Med. Aging 2020, 4, 38-44. [CrossRef] 
4. Spensley, M.; Del Borrello, S.; Pajkic, D.; Fraser, A.G. Acute Effects of Drugs on Caenorhabditis elegans Movement Reveal Complex Responses and Plasticity. G3 Genes Genomes Genet. 2018, 8, 2941-2952. [CrossRef]

5. Puchalt, J.C.; Sánchez-Salmerón, A.J.; Ivorra, E.; Genovés Martínez, S.; Martínez, R.; Martorell Guerola, P. Improving lifespan automation for Caenorhabditis elegans by using image processing and a post-processing adaptive data filter. Sci. Rep. 2020, 10, 8729. [CrossRef]

6. Jung, S.K.; Aleman Meza, B.; Riepe, C.; Zhong, W. QuantWorm: A Comprehensive Software Package for Caenorhabditis elegans Phenotypic Assays. PLoS ONE 2014, 9, e84830. [CrossRef]

7. Puchalt, J.C.; Layana Castro, P.E.; Sánchez-Salmerón, A.J. Reducing Results Variance in Lifespan Machines: An Analysis of the Influence of Vibrotaxis on Wild-Type Caenorhabditis elegans for the Death Criterion. Sensors 2020, 20, 5981. [CrossRef]

8. Sokolowski, M.B. Social Interactions in "Simple" Model Systems. Neurone 2010, 65, 780-794. [CrossRef]

9. De Bono, M.; Bargmann, C.I. Natural Variation in a Neuropeptide Y Receptor Homolog Modifies Social Behavior and Food Response in C. elegans. Cell 1998, 94, 679-689. [CrossRef]

10. Srinivasan, J.; Von Reuss, S.H.; Bose, N.; Zaslaver, A.; Mahanti, P.; Margaret, M.C.; O’Doherty, O.G.; Edison, A.S.; Sternberg, P.W.; Schroeder, F.C. A Modular Library of Small Molecule Signals Regulates Social Behaviors in Caenorhabditis elegans. PLoS Biol. 2012, 10, e1001237. [CrossRef]

11. Rogers, C.; Persson, A.; Cheung, B.; De Bono, M. Behavioral Motifs and Neural Pathways Coordinating O2 Responses and Aggregation in C. elegans. Curr. Biol. 2006, 16, 649-659. [CrossRef]

12. Dunsenbery, D.B. Video camera-computer tracking of nematode Caenorhabditis elegans to record behavioral responses. J. Chem. Ecol. 1985, 11, 1239-1247. [CrossRef]

13. Restif, C.; Ibáñez Ventoso, C.; Vora, M.M.; Guo, S.; Metaxas, D.; Driscoll, M. CeleST: Computer Vision Software for Quantitative Analysis of C. elegans Swim Behavior Reveals Novel Features of Locomotion. PLoS ONE 2014, 10, e1003702. [CrossRef]

14. Swierczek, N.A.; Giles, A.C.; Rankin, C.H.; Kerr, R.A. High-throughput behavioral analysis in C. elegans. Nat. Methods 2011, 8, 592-598. [CrossRef]

15. Ramot, D.; Johnson, B.E.; Berry, B., Jr.; Carnell, L.; Goodman, M.B. The Parallel Worm Tracker: A Platform for Measuring Average Speed and Drug-Induced Paralysis in Nematodes. PLoS ONE 2008, 3, e2208. [CrossRef]

16. Dusenbery, D.B. Using a microcomputer and video camera to simultaneously track 25 animals. Comput. Biol. Med. 1985, 15, 169-175. [CrossRef]

17. Simonetta, S.H.; Golombek, D.A. An automated tracking system for Caenorhabditis elegans locomotor behavior and circadian studies application. J. Neurosci. Methods 2007, 161, 273-280. [CrossRef] [PubMed]

18. Jaensch, S.; Decker, M.; Hyman, A.A.; Myers, E.W. Automated tracking and analysis of centrosomes in early Caenorhabditis elegans embryos. Bioinformatics 2010, 26, i13-i20. [CrossRef]

19. Boyd, W.; Anderson, G.; Dusenbery, D.; Williams, P. Computer Tracking Method for Assessing Behavioral Changes in the Nematode Caenorhabditis elegans. ASTM Int. 2000, 9, 225-238. [CrossRef]

20. Dzyubachyk, O.; Jelier, R.; Lehner, B.; Niessen, W.; Meijering, E. Model-based approach for tracking embryogenesis in Caenorhabditis elegans fluorescence microscopy data. In Proceedings of the 2009 Annual International Conference of the IEEE Engineering in Medicine and Biology Society, Minneapolis, MN, USA, 3-6 September 2009; pp. 5356-5359. [CrossRef]

21. Lorimer, T.; Goodridge, R.; Bock, A.K.; Agarwal, V.; Saberski, E.; Sugihara, G.; Rikin, S.A. Tracking changes in behavioural dynamics using prediction error. PLOS ONE 2021, 16, e0251053. [CrossRef]

22. Javer, A.; Currie, M.; Lee, C.; Hokanson, J.; Li, K.; Martineau, C.N.; Yemini, E.; Grundy, L.; Li, C.; Ch'ng, Q.; et al. An open-source platform for analyzing and sharing worm-behavior data. Nat. Methods 2018, 15, 645-646. [CrossRef]

23. Koopman, M.; Peter, Q.; Seinstra, R.I.; Perni, M.; Vendruscolo, M.; Dobson, C.; Knowles, T.; Nollen, E. Assessing motor-related phenotypes of Caenorhabditis elegans with the wide field-of-view nematode tracking platform. Nat. Protoc. 2018, 15, 2071-2106. [CrossRef]

24. Leonard, N.; Vidal-Gadea, A.G. Affordable Caenorhabditis elegans tracking system for classroom use. MicroPubl. Biol. 2021. [CrossRef]

25. Wählby, C.; Kamentsky, L.; Liu, Z.; Riklin Raviv, T.; Conery, A.; O’Rourke, E.; Sokolnicki, K.; Visvikis, O.; Ljosa, V.; Irazoqui, J.; et al. An image analysis toolbox for high-throughput C. elegans assays. Nat. Methods 2012, 9, 714-716. [CrossRef]

26. Rizvandi, N.B.; Pižurica, A.; Rooms, F.; Philips, W. Skeleton analysis of population images for detection of isolated and overlapped nematode C. elegans. In Proceedings of the 2008 16th European Signal Processing Conference, Lausanne, Switzerland, 25-29 August 2008; pp.1-5.

27. Rizvandi, N.B.; Pižurica, A.; Philips, W. Machine vision detection of isolated and overlapped nematode worms using skeleton analysis. In Proceedings of the 2008 15th IEEE International Conference on Image Processing, San Diego, CA, USA, 12-15 October 2008; pp. 2972-2975. [CrossRef]

28. Winter, P.B.; Brielmann, R.M.; Timkovich, N.P.; Navarro, H.T.; Teixeira Castro, A.; Morimoto, R.I.; Amaral, L.A.N. A network approach to discerning the identities of C. elegans in a free moving population. Sci. Rep. 2016, 10, 34859. [CrossRef] [PubMed]

29. Huang, K.M.; Cosman, P.; Schafer, W.R. Machine vision based detection of omega bends and reversals in C. elegans. J. Neurosci. Methods 2006, 158, 323-336. [CrossRef] [PubMed] 
30. Fontaine, E.; Burdick, J.; Barr, A. Automated Tracking of Multiple C. elegans. In Proceedings of the 2006 International Conference of the IEEE Engineering in Medicine and Biology Society, New York, NY, USA, 30 August-3 September 2006; pp. 3716-3719. [CrossRef]

31. Roussel, N.; Morton, C.A.; Finger, F.P.; Roysam, B. A Computational Model for C. elegans Locomotory Behavior: Application to Multiworm Tracking. IEEE Trans. Biomed. Eng. 2007, 54, 1786-1797. [CrossRef] [PubMed]

32. Nagy, S.; Goessling, M.; Amit, Y.; Biron, D. A Generative Statistical Algorithm for Automatic Detection of Complex Postures. PLoS Comput. Biol. 2015, 11, e1004517. [CrossRef] [PubMed]

33. Uhlmann, V.; Unser, M. Tip-seeking active contours for bioimage segmentation. In Proceedings of the 2015 IEEE 12 th International Symposium on Biomedical Imaging (ISBI), Brooklyn, NY, USA, 16-19 April 2015; pp. 544-547. [CrossRef]

34. Kiel, M.; Berh, D.; Daniel, J.; Otto, N.; Steege, A.T.; Jiang, X.; Liebau, E.; Risse, B. A Multi-Purpose Worm Tracker Based on FIM. bioRxiv 2018. [CrossRef]

35. Layana Castro, P.E.; Puchalt, J.C.; Sánchez Salmerón, A.J. Improving skeleton algorithm for helping Caenorhabditis elegans trackers. Sci. Rep. 2020, 10, 22247. [CrossRef] [PubMed]

36. Puchalt, J.C.; Sánchez-Salmerón, A.J.; Martorell Guerola, P.; Ivorra, E.; Genovés Martínez, S. Active backlight for automating visual monitoring: An analysis of a lighting control technique for Caenorhabditis elegans cultured on standard Petri plates. PLoS ONE 2019, 14, e0215548. [CrossRef]

37. Puchalt, J.C.; Sánchez-Salmerón, A.J.; Ivorra, E. Small flexible automated system for monitoring Caenorhabditis elegans lifespan based on active vision and image processing techniques. Sci. Rep. 2021, 11, 12289. [CrossRef] [PubMed]

38. Stiernagle, T. Maintenance of C. elegans. In C. elegans a Practical Approach; Oxford University Press: New York, NY, USA, 2006; pp. 2005-2018. [CrossRef]

39. Russ, J.C.; Neal, F.B. The Image Processing Handbook. In The Image Processing Handbook, 7th ed.; CRC Press, Inc.: Boca Raton, FL, USA, 2015; pp. 479-492.

40. Sossa, J.H. An improved parallel algorithm for thinning digital patterns. Pattern Recognit. Lett. 1989, 10, 77-80. [CrossRef]

41. Koul, A.; Ganju, S.; Kasam, M. Building The Purrfect Cat Locator App with TensorFlow Object Detection API. In Practical Deep Learning for Cloud, Mobile, and Edge: Real-World AI E Computer-Vision Projects Using Python, Keras E TensorFlow; O’Reilly Media: Sebastopol, CA, USA, 2019; Chapter 14, pp. 679-680 